\title{
Numerical investigation on vortex-induced motion of a pivoted cylindrical body in uniform flow
}

\author{
H. G. Sung ${ }^{1}$, H. Baek ${ }^{2}$, S. Hong ${ }^{1} \&$ J.-S. Choi ${ }^{1}$ \\ ${ }^{I}$ Maritime and Ocean Engineering Research Institute (MOERI; formerly \\ KRISO), KIOST (formerly KORDI), Daejeon, Korea \\ ${ }^{2}$ Department of Mathematics, MIT, Cambridge, USA
}

\begin{abstract}
Based upon our previous study presented in FSI 2011 ("Numerical simulation of vortex-induced vibration of a pivoted cylinder using the spectral element method", Fluid Structure Interaction, 2011), we demonstrate by numerical computation the effect of cross-sectional shape of cylinder which is pivoted at the wake of the body for the energy extraction via enhancement of VIV motion. The fluid equation is solved by using a computer code based upon the spectral element method (SEM) with the help of the arbitrary Lagrangian-Eulerian (ALE) framework. At first, the circular shape is tested, and then ellipses, whose ratios of the minor axis to the major axis are 0.6 and 0.8 , are compared with the case of the circular shape. We have found that ellipses enlarge displacements almost two times at Reynolds number 500. It was also found that increased drag forces contribute positively to the excitation torques.
\end{abstract}

Keywords: VIV, energy extraction, pivoted cylinder, Spectral Element Method (SEM), coupling of body motion with flow.

\section{Introduction}

In general, the vortex-induced-vibration (VIV) problem is regarded as a phenomenon to be avoided and reduced as noted in Blevins [2] and Sarpkaya [3]. As recently indicated by Raghavan [4], and Bernitsas and Raghavan [5], however, it seems to be reasonable to extract effective energy from the VIV phenomena. For example, our previous paper (Sung et al. [1]) demonstrated a meaningful trial in this direction by using pivoted circular cylinder subject to 
background uniform flows, which was motivated by Professor Bernitsas' researches (Bernitsas et al. [6]).

The present paper consists as follows. The governing equations are introduced and numerical methods employed in this study are explained in Section 2. Numerical results including comparison of forces and VIV among three different of cylinders are given in Section 3. Section 4 presents the meaning of the results for designing energy extraction devices by using a pivoted cylinder when the pivot point is at the wake of the body. In Section 5, concluding remarks with summary are suggested.

\section{Physical problem and numerical method}

As shown in Fig. 1, a submerged cylindrical body is pivoted to a column and exposed to VIV due to background fluid flow such as ocean currents. Rotation vector of the body can be denoted by $\vec{\Omega}(t)=(0,0, \theta(t))$ for the present case of $2 \mathrm{D}$. So, the angular velocity and acceleration can be obtained by time derivative of the rotation vector as $\dot{\vec{\Omega}}(t)$ and $\ddot{\vec{\Omega}}(t)$, respectively. We also assume the flow domain has a rectangular shape, and there is no free surface.
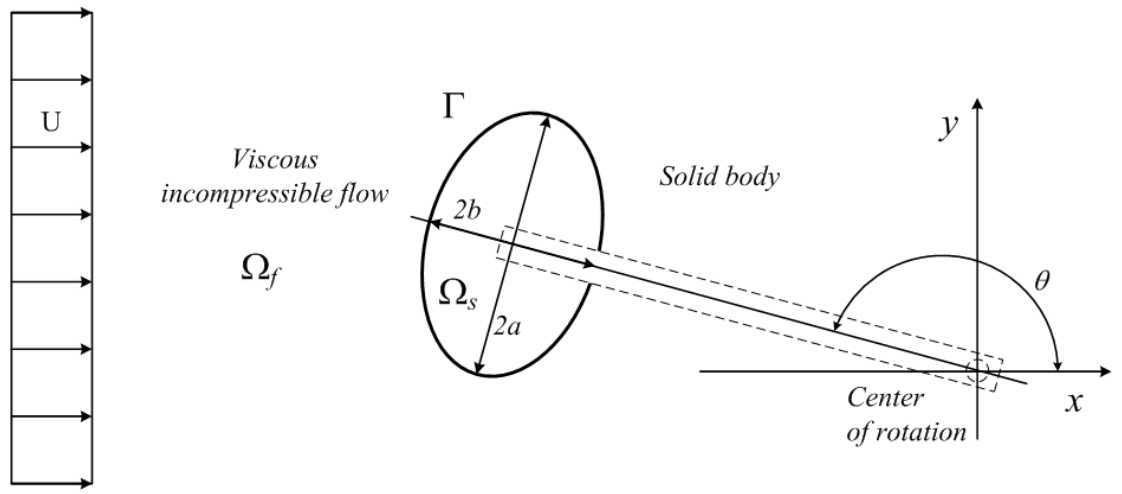

Figure 1: A schematic diagram of the VIV problem of a pivoted circular cylinder subject to uniform flow.

Details of the numerical method are referred to our previous paper (Sung et al. [1]), and here the brief summary of the numerical method is given. We describe the present flow problem with body motion by using the viscous incompressible flow model and ALE framework. The fluid equation is solved by using SEM -based computer code, NEKTAR which was originally developed by Professor Karniadakis at Brown University (Karniadakis and Sherwin [7]). The primary reason for using this code is due to necessity of attaining the high-order accuracy and robustness in time. The stiffly-stable time integration scheme utilized in this code is known to be very accurate and stable. Particularly in SEM, the condition number is very large compared with other discretization 
schemes and so the conventional time integration scheme such as the AdamsBashforth and Runge-Kutta methods may give rise to numerical instability.

The equation of body motion is modeled with a mass-spring-damper system, which is a reasonably accurate approximation to the prototype case. The external torque due to fluid motion can be computed by using SEM while the fluid structure interaction (FSI) is implemented as follows.

(1) Solve the equation for the solid part with the last solutions of the fluid part.

(2) Obtain the angular displacement, velocity, and acceleration by using a pertinent time integration algorithm, for example, Newmark scheme.

(3) Calculate the translational velocity and acceleration for the next time-step on the body boundary and transfer those values to the flow solver.

(4) Solve the governing equation of fluid motion, and the corresponding mesh field.

(5) Compute the traction on the interface between the solid sub-domain and the fluid sub-domain and find the torque acting upon the body.

(6) Repeat steps 1 to 5 up to the termination time.

While the previous study of Sung et al. [8] is based upon explicit coupling, the present scheme of FSI coupling requires sub-iterations for continuity in both velocity and traction at the common interface (Baek [9], and Baek and Karniadakis [10]).

\section{Numerical results and discussion}

An example of the grid system is shown in Fig. 2. It is noted that a series of convergence and sensitivity tests have been performed. It is noted that all simulations were carried upon in 2D because simulations in $3 \mathrm{D}$ are extremely

(a)

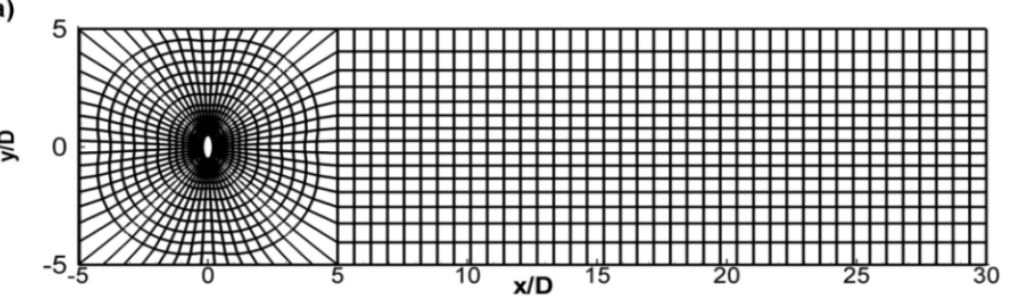

(b)

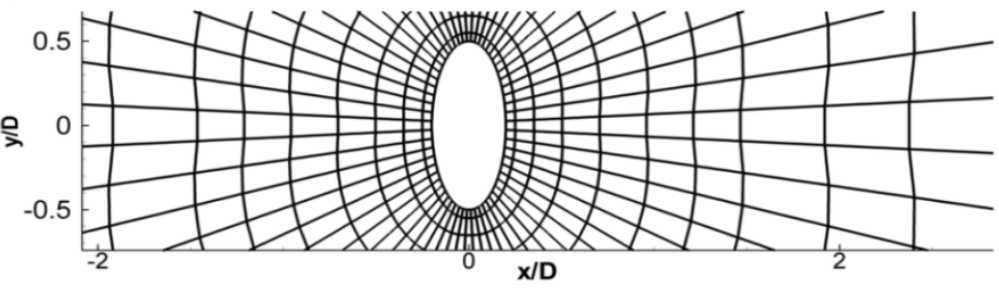

Figure 2: (a) Computational domain around a cylinder; (b) An enlarged plot around an elliptic cylinder. 
more expensive than $2 \mathrm{D}$ cases for our parametric study. However, we have to understand that 2D simulations of a cylinder are known to tend to overpredict the mean drag and root mean square (RMS) lift coefficients in general, as indicated by Persillon and Braza [11] and references therein, but they tend to underpredict the VIV amplitude, as seen in Evangelinos and Karniadakis [12].

Before we investigate the pivoted case, numerical results of stationary circular and elliptical cylinders case are given in very brief as follows. Details of the results can be referred to Sung et al. [8].

The cylinders used in the study have three shapes: circular (case 'ST1'), vertical ellipse of ratio 0.8 (case 'ST2'), and vertical ellipse of ratio 0.6 (case 'ST3'). For shorter and simple notation, we call an ellipse 'parallel (vertical)' when the major axis is parallel (vertical) to the incoming flow, respectively. By analyzing drag and lift force history of fixed cylinders with different shape of cross section, it is found out to our expectation that the drag forces increase as the ratio of the minor axis to the major axis decreases as shown in Fig. 3. It is not clear whether the lift force would increase because the projected surface area decreases.
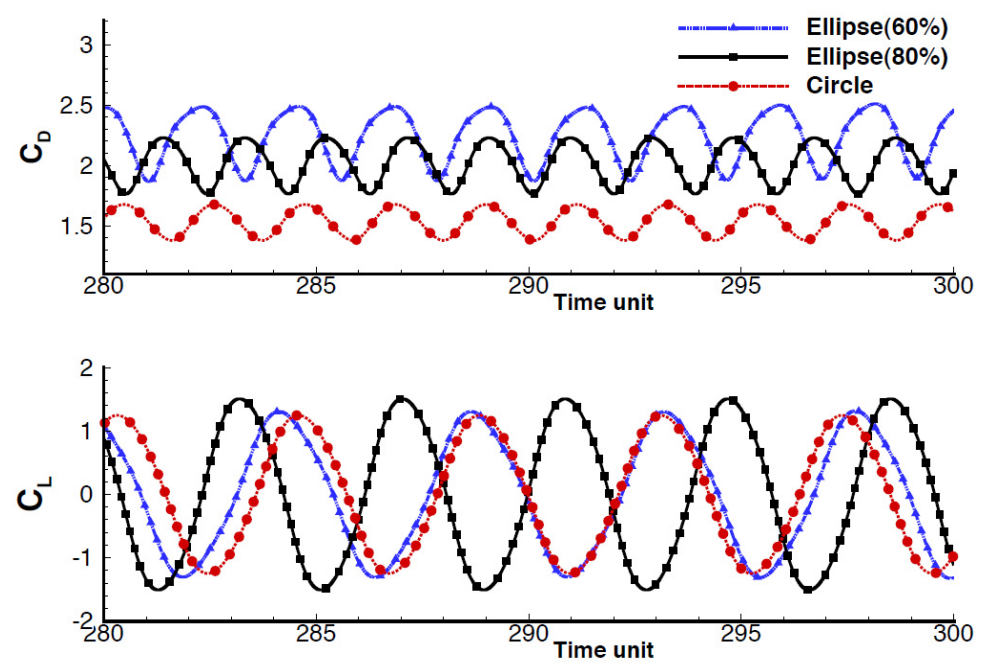

Figure 3: Comparison of the lift and drag coefficients among cylinders with different ratios, i.e., Cases 'ST1' - Circle, 'ST2' - Ellipse (80\%), 'ST3' - Ellipse (60\%).

Numerical results of stationary circular and elliptic cylinders at Reynolds number 500 are listed in Table 1. As the ratio b/a gets smaller from 1.0 to 0.6 , the shedding frequency $f_{0}$ changes slightly up to $12 \%$. It is found that their odd multiple harmonics $3 f_{0}, 5 f_{0}$, and $7 f_{0}$ can clearly be seen. In case 'ST3', i.e. $60 \%$ vertical ellipse, the lift forces are not steady but there is a small modulation of amplitude while other two cases show a lot more steady oscillation. The drag force gets larger as the ratio b/a gets smaller as expected due to the pressure 
decrease in the wake. The lift force, however, cannot increase because of the smaller projection area onto a streamwise plane. The drag and lift force coefficients use the diameter of cylinder or ellipse along the major axis. The vorticity fields of cases 'ST2' and 'ST3' show a much more irregular pattern of vorticity in the wake than that of case 'ST1'. It is also confirmed that shedding vortices are placed in a straight line in the wake in case 'ST1' while cases 'ST2' and 'ST3' show more chaotic vortex shedding.

Table 1: Peaks of the power spectrum of the lift force at $\mathrm{Re}=500$ for stationary cylinders. Numbers in parentheses are magnitudes in log scale at the corresponding frequency.

\begin{tabular}{|c|c|c|c|c|c|}
\hline Cases & $\mathrm{b} / \mathrm{a}$ & $f_{1}$ & $f_{2}$ & $f_{3}$ & $f_{4}$ \\
\hline ST1 & 1.00 & $0.234(4.76)$ & $0.703(1.68)$ & $1.173(-0.36)$ & $1.639(-2.36)$ \\
\hline ST2 & 0.80 & $0.263(5.46)$ & $0.780(2.59)$ & $1.308(0.71)$ & $1.825(-1.21)$ \\
\hline ST3 & 0.60 & $0.222(5.14)$ & $0.667(2.71)$ & $1.108(0.25)$ & $1.559(-2.75)$ \\
\hline
\end{tabular}

Now, results of VIV motion of a pivoted cylinder are presented. In cross flow VIV where streamwise motion is prohibited (most of the cases in this field of study), drag force does not affect the motion at all. When a rigid body is pivoted, however, VIV is expected to depend on the configuration, i.e. whether the pivot is located at an upstream or downstream location. For cases with the arm length, $r=3$, we compare the effect of cross-sectional shape of cylinders on VIV motion. The effect of the arm length is elaborated in detail in Sung et al. [8].

The four cases from 'PC0' to 'PC3' are listed in Table 2 with RMS angular displacements and velocity. The spring constant is also fixed with the natural frequency $2 \pi \times 0.24$, which is close to the vortex shedding frequency in the stationary circular cylinder at $\mathrm{Re}=500$. The time series of the angular displacement of 'PC0', 'PC1', 'PC2', and 'PC3' are shown in Fig. 4. Case 'PC2' shows amplitude modulation slightly while other cases do not. The frequency spectrum analysis indicates the cylinders are locked in, i.e., the dominant frequency of motion is close to the vortex shedding frequency.

Table 2: $\quad$ RMS angular displacement and velocity of pivoted cylinders with arm length 3 and different shapes at $\mathrm{Re}=500$. The natural frequency $\omega$ is set to $2 \pi \times 0.24$. $r$ and $J$ are the arm length and the moment of inertia, respectively.

\begin{tabular}{|c|c|c|c|c|c|c|}
\hline Cases & $\begin{array}{c}\text { Minor } \\
\text { axis, a }\end{array}$ & $\begin{array}{c}\text { Major } \\
\text { axis, b }\end{array}$ & $r$ & $J$ & $\theta_{r m s}$ & $\dot{\theta}_{r m s}$ \\
\hline PC0 & 0.5 & 0.5 & 3.0 & 46.01 & 0.0768 & 0.1096 \\
\hline PC1 & 0.5 & 0.5 & 3.0 & 36.79 & 0.0838 & 0.1217 \\
\hline PC2 & 0.4 & 0.5 & 3.0 & 36.79 & 0.1196 & 0.1570 \\
\hline PC3 & 0.3 & 0.5 & 3.0 & 36.79 & 0.1623 & 0.2415 \\
\hline
\end{tabular}


Now, we show how a natural frequency of cylinders affect the motion and investigate whether the elliptic cylinders demonstrate larger VIV motion over wide range of reduced velocities. The tested reduced velocities are $\mathrm{V}_{\mathrm{r}}=2.32$, 2.70, 3.33, 4.16, 4.76, 5.55, and 8.33.

The angular displacement are plotted against the reduced velocity $\mathrm{V}_{\mathrm{r}}=\mathrm{D} / \mathrm{Uf}$ in Fig. 5, where $\mathrm{D}, \mathrm{U}$, and $\mathrm{f}$ are the semi major axis $(0.5)$, the incoming fluid

(a)

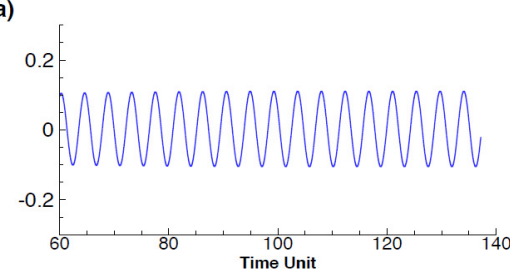

(c)

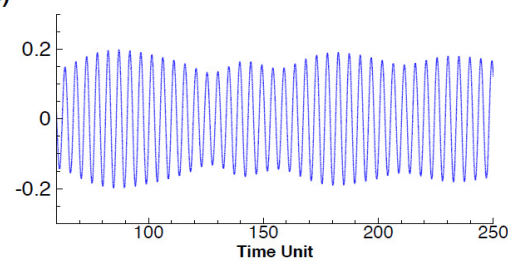

(b)

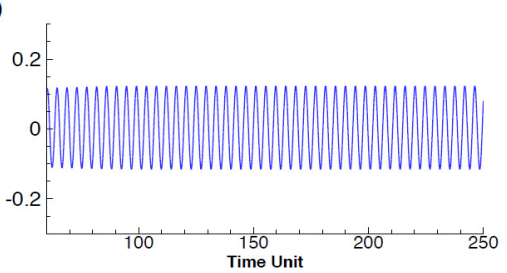

(d)

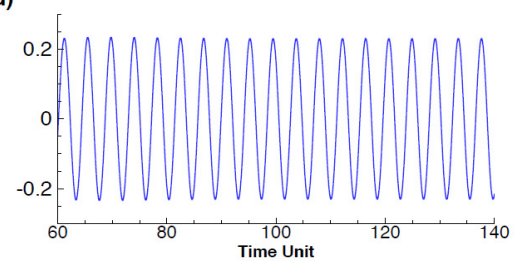

Figure 4: Angular displacement of cases (a) PC0, (b) PC1, (c) PC2, and (d) PC3.
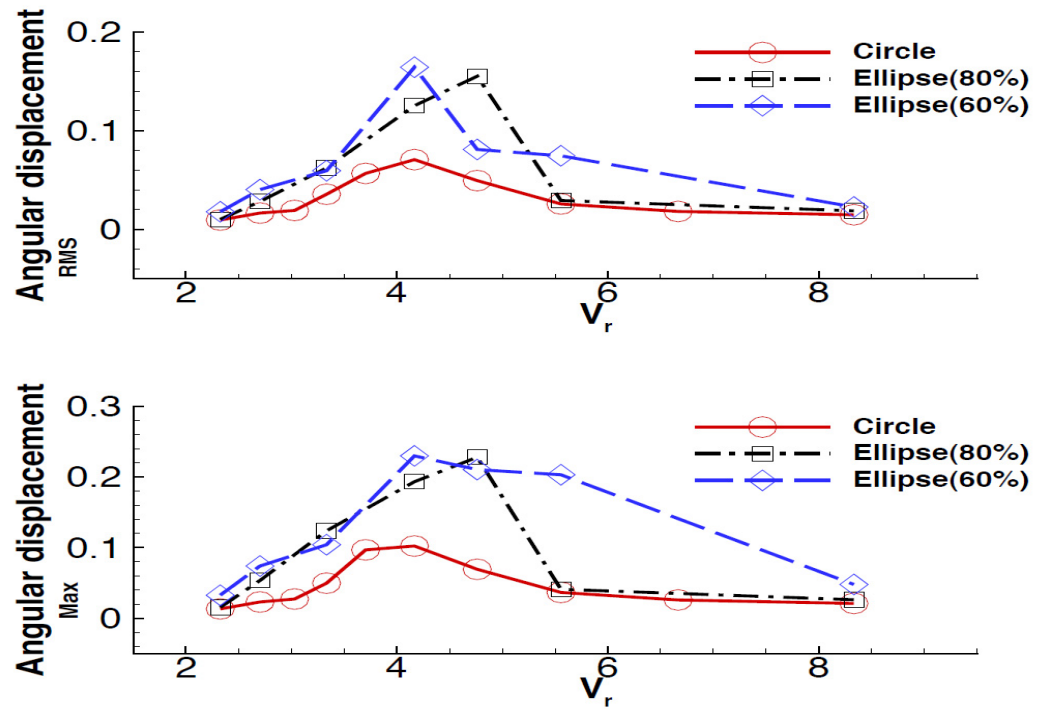

Figure 5: $\quad$ RMS (top) and maximum (bottom) angular displacement versus reduced velocity of cases 'PC1', 'PC2', and 'PC3'. 
velocity (1.0), and the natural frequency of the attached spring. Case 'PC3', i.e. $60 \%$ vertical ellipse, performs best among three cases over the wide range of reduced velocity. The peak angular displacements of cases 'PC1', 'PC2', 'PC3' are $0.1023\left(\mathrm{~V}_{\mathrm{r}}=1 / 0.24\right), 0.228\left(\mathrm{~V}_{\mathrm{r}}=1 / 0.21\right), 0.2299\left(\mathrm{~V}_{\mathrm{r}}=1 / 0.24\right)$, respectively.

Due to the modulation in the displacement, RMS is plotted as well as the maximum amplitude. In both plots, $60 \%$ vertical ellipse shows two times larger amplitude than a corresponding cylinder. The peak RMS angular displacement occurs at different reduced velocity for cases 'PC2' and 'PC3'. The natural frequencies of spring at the peak RMS displacement are in good agreement with the vortex shedding frequencies of stationary cylinders. More specifically, as listed in Table 1, the dominant shedding frequencies are $0.234,0.263$, and 0.222 for cases 'ST1', 'ST2', and 'ST3', respectively, while the peak RMS angular displacements are observed at frequencies $0.24,0.21$, and 0.24 for corresponding cylinders.

\section{Summary and conclusion}

Numerical simulation of VIV motion of pivoted cylinders was described. The cylinders are subject to steady uniform flow when the pivot is placed at a downstream location and vary from a circle to ellipses. The present flow model and method utilizes an SEM-based N-S solver, which was originally developed by Professor G.E. Karniadakis' group. The present method of coupling of the body motion with the flow problem is based upon a strong coupling scheme. Through a series of numerical simulation, it was demonstrated that drag forces increase leading to larger displacements for elliptical cylinder instead of circular shape, while the lift forces decrease. Practical questions such as feasibility of any energy extraction devices utilizing the present concept and comparison of efficiency of pivoted cylinders with that of linearly moving cylinders need to be studied in the near future.

\section{Acknowledgements}

The present study is supported by the "Development of Key-Technology of Ocean Renewable Energy using Vortex-Induced Vibration (VIV) in Water Flow" granted by the Ministry of Knowledge Economy of Korea and the principal R\&D program of KIOST: "Performance Evaluation Technologies of Offshore Operability for Transport and Installation of Deep-sea Offshore Structures" granted by Korea Research Council of Public Science and Technology. The authors express deep gratitude to Professor Karniadakis for permission of the computer code, known as NEKTAR. 


\section{References}

[1] HG Sung, H Baek, S. Hong, J-S. Choi, "Numerical simulation of vortexinduced vibration of a pivoted cylinder using the spectral element method", Fluid Structure Interaction, 2011.

[2] Blevins, R. D. Flow-Induced Vibration, Malabar, FL Krieger Publishing Company, 1994.

[3] Sarpkaya, T. A critical review of the intrinsic nature of vortex-induced vibrations, Journal of Fluids and Structures, Vol. 19, pp. 389-447, 2004.

[4] Raghavan, K, "Energy Extraction from a Steady Flow Using Vortex Induced Vibration”, Ph.D. Dissertation, The University of Michigan, 2007.

[5] Bernitsas, M.M. and Raghavan, K. "Reduction/Suppression of VIV of Circular Cylinders through Roughness Distribution at $8 \times 10^{3}<\operatorname{Re}<1.5 \times 10^{5}$, , Proc. OMAE Conference, pp. 1-5, 2008.

[6] Bernitsas, M.M., Raghavan, K., Ben-Simon, Y. and Garcia, E.M.H. "VIVACE (Vortex Induced Vibration for Aquatic Clean Energy): A New Concept in Generation of Clean and Renewable Energy from Fluid Flow", Proc. OMAE Conference, pp. 1-18, 2006.

[7] Karniadakis, D., and S.J. Sherwin, Spectral/hp Element Methods for CFD, Oxford University Press, $2^{\text {nd }}$ Ed., London, 2005.

[8] HG Sung, H Baek, S. Hong, J-S. Choi, "Vortex-induced vibration enhancement for harvesting energy in currents using a pivoted elliptic cylinder”, J. Fluid \& Structure (Submitted), 2012.

[9] Baek, H. 2010, A spectral element method for fluid-structure interaction: new algorithm and application to intracranial aneurysms. Ph.D. Thesis, Brown University.

[10] Baek, H. and G.E. Karniadakis, 2010, Stabilization of fluid-structure interactions via fictitious added mass and damping in physical and biological applications, submitted to Elsevier.

[11] Persillon, H., Braza, M., "Physical analysis of the transition to turbulence in the wake of a circular cylinder by three-dimensional Navier-Stokes simulation", J. Fluid Mech. 365, 23-88, 1998.

[12] Evangelinos, C., Karniadakis, G. E., "Dynamics and flow structures in the turbulent wake of rigid and flexible cylinders subject to vortex-induced vibrations" J. Fluid Mech. 400, 91-124, 1999. 